\title{
Central pontine myelinolysis
}

S K Chamarthi
Department of Radiodiagnosis, Apollo Hospitals, Narehenpita,
Colombo, Sri-Lanka
A L Kiranmayi
A M Mukarrab

\section{Introduction}

Central pontine myelinolysis is a demyelinating disease of the pons characterised by loss of myelin and oligodendroglia with relative neuron sparing. This condition classically occurs in alcoholics, malnourished or chronically debilitated adults ${ }^{1}$ and usually occurs in the hospital setting, a few days following rapid correction of hyponatraemia. We report a case of central pontine myelinolysis in a chronic alcoholic, malnourished patient following rapid correction of hyponatraemia.

\section{Case report}

A 44-year-old man presented to the emergency department with generalised weakness and altered speech of 1 week's duration. There was a history of an accidental fall at home with scalp injury. The patient was known to have alcoholic liver disease. On clinical examination the patient was found to be malnourished and anaemic. The liver was palpable. Serum electrolyte analysis revealed hypokalaemia $(2 \mathrm{meq} / \mathrm{l})$ and hyponatraemia (10 meq /l). Ultrasound scan of the abdomen showed an enlarged liver with diffuse fatty infiltration. Computed tomography (CT) scan of the brain was normal. Electrolyte imbalance was corrected using potassium chloride in dextrose saline and 3\% normal saline. Following this treatment the patient improved and was discharged from the hospital.

One week later he presented again with confusion and altered levels of consciousness. Repeat CT scan of brain was normal. The patient was then sent for magnetic resonance imaging (MRI) examination of the brain. MRI was done using a 3T magnet. Scanning was performed in the three orthogonal planes and diffusion imaging/apparent diffusion coefficient (ADC) mapping was also done.

MRI images revealed an area of altered signal intensity in the pons, predominantly central, with sparing of the periphery. The lesion was hypointense on T1-weighted images (T1WI) and hyperintense on T2WI (Figs 1 and 2), FLAIR and diffusion images. ADC mapping demonstrated low signal in the pons. There was predominant involvement of the transverse pontine fibres, with relative sparing of the descending corticospinal tracts. The rest of the brain parenchyma showed normal signal intensity. The patient's condition gradually deteriorated further, with development of quadriparesis and pseudobulbar palsy due to the involvement of the corticospinal and corticobulbar tracts.

\section{Discussion}

Central pontine myelinolysis is a non-inflammatory demyelinating disease characterised by loss of myelin with relative neuron sparing. It may be associated with demyelination elsewhere in the central nervous

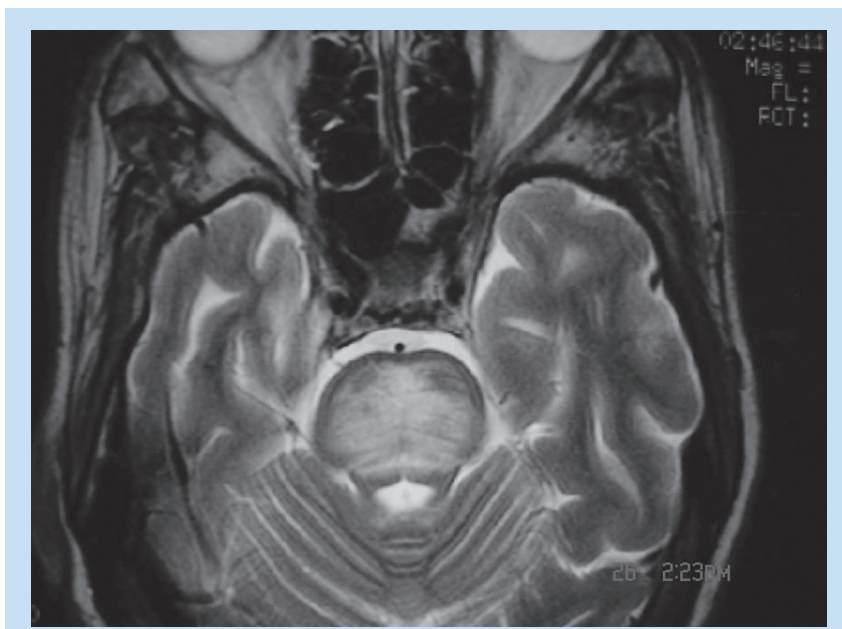

Fig. 1. Axial-T2W MR scan showing hyperintense signal in the central pons with predominant involvement of transverse pontine fibres and relative sparing of descending corticospinal tracts.

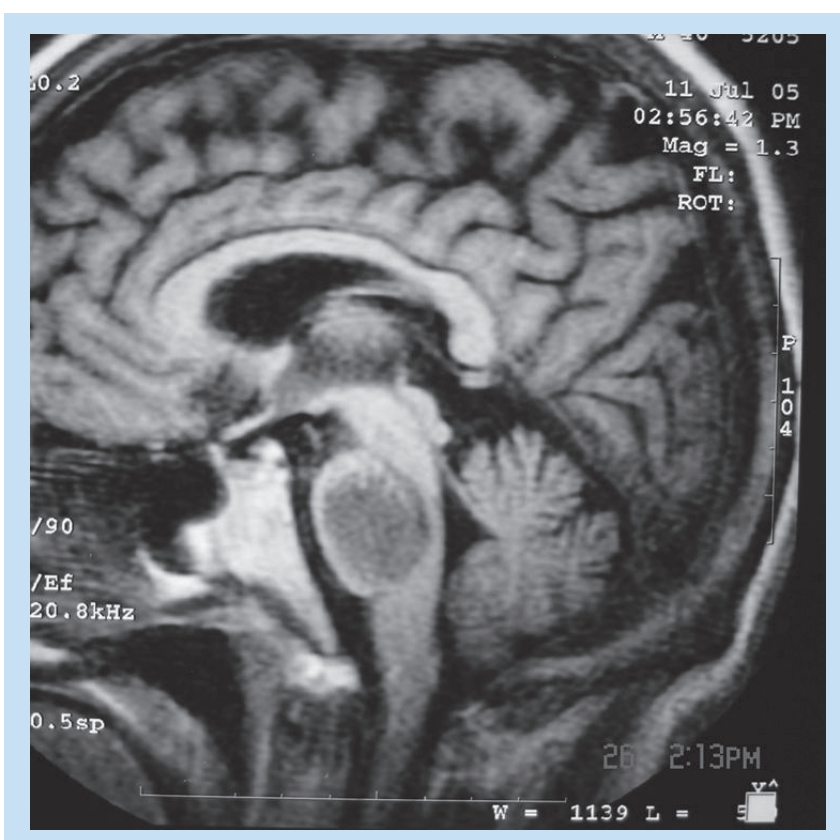

Fig. 2. Sagittal T1WI showing hypointense lesion in the central pons.

system. The common extrapontine sites are the putamen, caudate nucleus, midbrain, thalamus and subcortical white matter (extrapontine myelinolysis). ${ }^{2}$ Over $75 \%$ of cases are associated with chronic alcoholism or rapid correction of hyponaetraemia and sometimes hypernaetraemia. ${ }^{3,4}$ A good correlation was found between development of central pontine myelinolysis and the correction of sodium more rapidly than $12 \mathrm{meq} /$ day. ${ }^{5}$ A number of other conditions have been associated with central pontine myelinolysis such as orthotopic liver transplantation, chronic renal failure, dehydration, diabetes mellitus, sepsis, advanced malignancy and acute haemorrhagic pancreatitis. ${ }^{5}$ 
The pathophysiological mechanism is unknown. Since both the pontine and extrapontine sites involved have a rich grey and white matter interface, it has been hypothesised that a rapid osmotic change causes an endothelial injury in the more vascular grey and white matter which induces the release of myelinolytic factors that damage the adjacent white matter. ${ }^{6}$ The term osmotic myelinolysis is currently favoured for this condition.

The initial symptoms may be weakness, confusion and dysarthria. In severe cases there is spastic quadriparesis and pseudobulbar palsy. This may evolve within 3 - 10 days into a locked-in-state (pseudo coma). Death within 2 - 3 months is the usual outcome. The 6-month survival rate is $5-10 \% .^{5}$

The imaging features are due to increased water content in the affected areas. Transverse pontine fibres are more severely affected, compared with descending corticospinal tracts. NECT scans are normal or disclose nonspecific hypodense areas. Lesions appear hypointense on T1WI and hyperintense on T2WI and show varied enhancement following contrast administration. ${ }^{6}$

Differential diagnosis of central pontine myelinolysis includes infarct, metastasis, glioma, multiple sclerosis, encephalitis, radiotherapy and chemotherapy. ${ }^{3}$ However concomitant involvement of the pons and basal ganglia is specific for osmotic myelinolysis. ${ }^{7}$ In such cases the imaging differential diagnosis includes hypoxia, Leigh disease and Wilson's disease. The classical history combined with imaging findings distinguishes osmotic myelinolysis from the rest of the conditions.

A number of therapeutic approaches have been tested, although no specific therapy exists. Correction of serum sodium should not exceed $12 \mathrm{meq} / 24 \mathrm{~h}$. Recovery varies from no improvement to substantial recovery, although the outcome of this condition is frequently fatal.

1. Ho VB, Fitz CR, Yoder CC, Geyer CA: Resolving MR features in osmotic myelinolysis (central pontine and extrapontine myelinolysis). Am J Neuroradiol 1993; 14:163-167.

2. Koci TM, Chiang F, Chow P, et al. Thalamic extrapontine lesions in central pontine myelinolysis. Am J Neuroradiol 1990;11: 1229-1233.

3. Miller GM, Baker HL jun.., Okozaki H, Whisnant JP. Central pontine myelinolysis and its imitators: MR findings. Radiology 1988; 168:795-802.

4. Clark WR. Diffuse demyelinating lesions of the brain after the rapid development of hypernaetremia. West J Med 1992;157:571-573.

Howard LS, Krishna Rao CVG, Zimmerman RA. Cranial MRI and CT. Mc Graw-Hill, 1999:606-608.

6. Koragi Y, Takahashi M, Shinzaho J, et al. MR findings in two presumed cases of mild central pontine myelinolysis, Am J Neuoradiol 1995;14:651-654.

7. Osborn AG. Diagnostic Neuroradiology. Mosby, 1994:761-763.

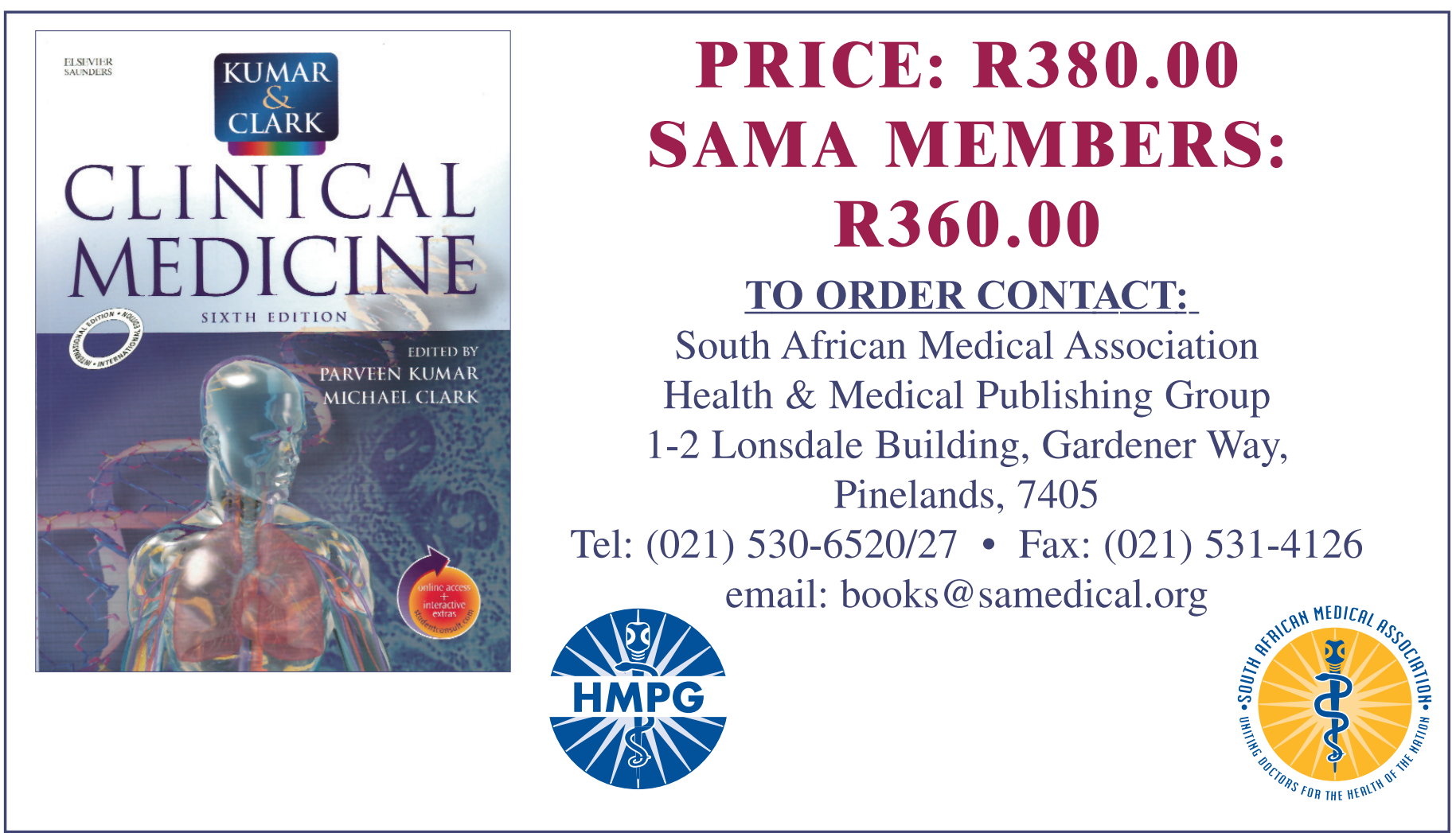

